Pfr. E. Rippmann, Zürich :

Dr. J. Gabus, Neuenburg:

Dr. E. Gerber, Schinznach :
Historische und archäologische Dokumente aus der Arabia petraea.

Vie sociale et vie psychique des Esquimaux cariboux.

Lage und Gliederung des Lauterbrunnentals.

\title{
EXKURSIONEN
}

1940/41 Moränenlandschaft Dietikon-Engstringen, Leiter Prof. Dr. O. Flückiger. Grüningen-Wetzikon, Leiter Prof. Dr. O. Flückiger.

1941/42 Rafzerfeld, Leiter Dr. H. Hofer.

1942/43 Glarnerland, Leiter Dr. R. Streiff-Becker.

Von Rikon nach Elgg, Leiter Prof. Dr. W. Wirth und Dr. P. Walther.

1943/44 Hoher Ron und Hüttner Seen, Leiter Dr. W. Höhn-Ochsner.

1944/45 Nußbaumer Seen, Leiter Prof. Dr. H. Boesch und Prof. Dr. A. U. Däniker.

\section{DER GEGENWÄRTIGE WANDEL ALPINER KULTURLANDSCHAFTEN}

\author{
HEINRICH GUTERSOHN
}

Unsere Zeit ist eine Zeit hektischer Veränderungen der Kulturlandschaften. Infolge der beschleunigten Bevölkerungszunahme, der anhaltenden wirtschaftlichen Konjunktur und der Konzentration der Wohnstätten wachsen die Großstädte rascher als je, entfalten sich aber auch bis anhin ländlich-ruhige Dorf- und Weilergruppen zu Agglomerationen und damit zu sekundären Ballungsräumen. Der Bedarf an Boden für Wohnund Industriebauten geht zu Lasten bisherigen Agrarlandes, macht aber auch vor dem verfassungsmäßig geschützten Waldbestand nicht halt, werden doch Teilstücke unseres werdenden Nationalstraßennetzes auch durch den Wald gezogen. Man hat den Eindruck, daß diese Entwicklungen überborden und dem Willen der Mehrheit unseres Volkes entgleiten; man ruft deshalb nach straffer Planung und nach bewußter Lenkung des Geschehens.

Das Gegenstück zu dieser Entwicklung ist die Rezession in den Alpentälern. Die bisherige Nutzung wird hier extensiviert, Fluren werden völlig aufgegeben, die bäuerliche Bevölkerung schwindet, Wohnstätten sind wüstgelegt und stehen in Verfall. Niemandem, der in unsern Berggebieten wandert, kann die schleichende, in ihren Folgen geradezu beängstigende Wandlung verborgen bleiben. Auf manchen Berghöfen arbeiten nur noch alte Leute, die jungen sind abgewandert. Mit Beklemmung erwartet man die kommenden Jahre, in denen unweigerlich viele weitere Bergbetriebe endgültig verlassen werden müssen.

\section{BEVÖLKERUNGSABNAHME UND EXTENSIVIERUNG DER NUTZUNG}

Die erwähnte Extensivierung manifestiert sich in verschiedener Weise. So wurden hochgelegene Kuhalpen zu Galtviehalpen, die Sennereien also stillgelegt. Freilich braucht diese Umstellung noch nicht nachteilig zu sein; denn Jungvieh zu sömmern, ist auf lange Sicht möglicherweise ebenso einträglich wie die Sömmerung der Milchtiere. Aber es handelt sich doch mindestens um eine örtliche Minderung der Produktion. Tiefgreifender aber ist die Umstellung von Kuhalpen zu Schafweiden. Die Bergalp im Meiental UR hat fünf Stafel; sie werden indessen seit 1959 nur noch als Schafweiden 
benützt. Zurückgegangen ist auch die Zahl der Ziegen und dementsprechend die der früheren Geißalpen. Im Kanton Glarus zählte man 18967040 Ziegen, 1956 noch 2333, in Engi GL waren es früher 1000, 1956 noch 252. Das schon von Stebler erwähnte, an der Hauptstraße in Engi stehende, aus vielen Ställen sich zusammensetzende Geißdörfchen wird nur noch mäßig, ein ähnliches, weiter taleinwärts bei Schwändi (Elm GL) gelegenes, überhaupt nicht mehr benützt. Die Geißhirten, welche in den Bergdörfern am frühen Morgen mit dem Horn ihre Ziegen zusammenriefen, mit ihnen auf die Alp stiegen und abends wieder heimkehrten, gehören vielerorts der Vergangenheit an. Anstelle der Ziegen werden Schafe gehalten, die einer speziellen Hut bedürfen.

Drastisch ist auch die Umwandlung von Äckern zu Wiesen, nur handelt es sich dabei um eine Änderung, die bereits vor Jahrzehnten, nämlich mit dem Aufkommen besserer Verkehrsverbindungen und entsprechend erleichterten Getreidezufuhren, einsetzte. Würde die Produktion nicht mit Anbau- und Mahlprämien gestützt, so müßten wohl weitere Bergäcker verschwinden. Auf vielen terrassierten ehemaligen Ackerparzellen wächst nur noch Futtergras.

Ortsbürgergemeinden oder Korporationen pflegen ihren Mitgliedern Allmendteile - Löser, Allmendgärten (UR), Saaten (GL) - zur Nutzung zuzuweisen. Früher wurden hier vorwiegend Gemüse gepflanzt. Leider aber werden manche dieser Parzellen nicht mehr bezogen und bepflanzt. Der Verzicht wird mit zu langen Arbeitswegen oder mit der Kleinheit des Landstückes begründet. Im Urnerland rückt die Korporation die wenigen noch in Anspruch genommenen Gärten zusammen und verpachtet die dadurch frei gewordenen und zusammengelegten äußeren als Wiesland. Schon unterbleibt gelegentlich die übliche Nutzung privaten Bodens in unmittelbarer Nähe der Dörfer. Im Sommer 1962 wurden zum Beispiel am Rande des Kurortes Schuls (GR) einzelne Fettwiesen nicht mehr gemäht.

Auch die Nutzungsrechte über besonders hoch gelegene Fluren werden immer weniger ausgeübt. Wildheuplanggen, die man früher zu verlosen pflegte, finden nur noch wenige Interessenten, und dasselbe gilt für manche Bergwiesen (Heuwiesen, Heumäder), obwohl sie eher günstiger gelegen und ertragreicher sind. Der völlige Verzicht auf die Nutzung bisheriger Alpweiden ist namentlich im Kanton Tessin alarmierend. Die Bestände an Rindvieh sanken hier von 43000 im Jahre 1896 auf 26800 im Jahre 1956. Man weiß, daß der Kanton Tessin im Jahre 1864 noch 558 Alpen zählte; 1956 waren ihrer 266 überhaupt nicht mehr bestoßen, und man geht wohl nicht fehl in der Annahme, daß während der vergangenen hundert Jahre die Hälfte der Tessiner Alpen aufgegeben und daher sukzessive von Busch überwuchert wurde, ein tiefgreifender wirtschaftlicher Wandel, ein Wandel auch der Landschaft, bei welchem sich aus einer einstigen, immerhin vielen Tessinern eine Existenzgrundlage bietenden Kulturlandschaft eine sekundäre Naturlandschaft zu entwickeln anhebt.

Parallel zu diesem Schwinden der Nutzung geht das stete Absinken der Dauersiedlungsgrenze, indem bisherige Berghöfe zu Maiensäßen oder überhaupt völlig aufgegeben werden, ein Vorgang, der sich in vielen Tälern und Beispielen belegen ließe. Gleichzeitig sinkt die Wohndichte in den Dörfern selber. Am stärksten in Mitleidenschaft gezogen werden natürlich die höchstgelegenen, die aus klimatischen Gründen ärmlichen, die verkehrsungünstigen Gebiete. Es sind ja zugleich die Gebiete, in denen die Arbeit ohnehin schwerer, die Erträgnisse zudem fast durchwegs dürftiger sind als in tieferen Lagen und namentlich als im Mittelland, ja wo Existenzgrundlagen vorwalten, die man heute kaum mehr jemandem zumuten kann. Von den 21 Gemeinden des tessinischen Bezirkes Leventina verzeichneten allein im Jahrzehnt 1950 bis 1960 ihrer 10 einen Rückgang der Einwohnerzahlen, und zwar waren darunter vier Gemeinden mit einem Verlust von mehr als 30\%! Ähnlich war es im Bezirk Locarno: In 28 von den total 45 Gemeinden nahm die Einwohnerzahl ab, in 9 Gemeinden um mehr als $30 \%$. In Rasa sank die Zahl der Bewohner von 38 auf 19, das heißt um 50\%. Ähn- 
liche, wenn auch nicht derart massierte Beispiele ließen sich von andern Bergkantonen nennen.

\section{KORREKTUREN DER ALPWIRTSCHAFT}

Nun ist aber dieser Rückgang von Einwohnerschaft, Siedlungseinheiten und Wirtschaftsflächen nicht nur negativ zu beurteilen. Wenn die allgemeinen Lebensbedingungen wesentlich härter sind als in andern Landesteilen, so kann sich die Abwanderung auch als notwendiger Weg zur Gesundung von Mensch und Wirtschaft erweisen. Das Verlassen von Häusern und Fluren ist vielerorts die unvermeidliche Voraussetzung für das Wohlergehen sowohl der Abgewanderten als auch der Zurückgebliebenen, denn diese sind nun weniger eingeengt, ihr Bergbauernbetrieb kann verbessert werden, und damit ist auch die Bahn frei für eine allgemeine Hebung der Lebenshaltung. Wahrscheinlich ist der heutige Rückgang vielerorts einfach die unerläßliche Korrektur für eine seinerzeit allzu weit getriebene Erschließung und Besetzung von Berggebieten. Diese Korrektur wird nun aber nicht nur durch die Abwanderung eines Teils der Bevölkerung, sondern gleichzeitig durch weitere positive Maßnahmen eingeleitet, auf die noch einzutreten sein wird. Es macht gegenwärtig den Anschein, als sei in den alpinen Gebieten ein Wettlauf im Gange: Einerseits lockt das Mittelland und lädt den Bergler ein, sein beschwerliches Dasein mit einem besseren zu vertauschen; anderseits aber beginnen eingeleitete und manche schon abgeschlossene Verbesserungen und Umstellungen sich günstig auszuwirken und einen Teil der Bergbevölkerung wieder fester an die Scholle zu binden. Die Berggebiete unterliegen also nicht nur der Abwanderung und der inneren Verarmung ihrer Betriebe, sondern manche machen einen bedeutenden Strukturwandel im Sinne einer wesentlichen Aufwertung durch. Daß damit nicht nur soziologische Umstellungen, sondern auch Wandlungen der Landschaft verbunden sind, liegt auf der Hand.

Die im Gang befindlichen und die bereits abgeschlossenen Korrekturen wurzeln in mancherlei günstigen Voraussetzungen. Zunächst ist die erfreuliche Tatsache der besseren Fachkenntnisse hervorzuheben, welche auf Forschung und Ausbildung sowohl der Ingenieur-Agronomen als auch vieler Besucher von Berufsschulen fußt. Bund und Kantone sowie Berufsorganisationen, wie der Schweizerische Bauernverband und die 1943 ins Leben gerufene Schweizerische Arbeitsgemeinschaft der Bergbauern, bemühen sich, auf dem Weg über Bestandesaufnahmen, Kredithilfen, Beratung und Schulung die Existenzgrundlagen der Bergbevölkerung allseitig zu stärken. In vielen Berggemeinden erwies sich der Bau von Kraftwerken als zunächst in ihrem Ausmaß unerwartete, große Hilfe, können doch auch hier manche Arbeitskräfte verwendet, namentlich aber auch die in Form von Konzessionsgebühren, Wasserzinsen und Steuern eingenommenen Gelder zugunsten dringender öffentlicher Aufgaben eingesetzt werden. Eine weitere wirksame Hilfe bringt manchem Alpwirtschaftsgebiet der Fremdenverkehr, indem er Arbeits- und Absatzmöglichkeiten sowie größere Steuereingänge bringt, die andere Bergzonen nicht kennen. In einigen Walliser Gemeinden waren Bestrebungen zur Heranziehung von Industrien von Erfolg gekrönt ; nicht nur Männer, sondern auch Frauen und Töchter finden nun eine lohnende Beschäftigung an der Werkbank.

Im folgenden seien einige dieser positiven Wandlungen anhand von Beispielen erörtert.

\section{VERBESSERUNG VON BETRIEBSFORM UND BETRIEBSSTRUKTUR}

Eine erste Gruppe von Maßnahmen sind solche, die der Verbesserung der Betriebsform und -struktur dienen, wie die Umstellung von Einzel- auf Genossenschaftsalpung, die Konzentration von Verarbeitungsstätten, die Aufhebung unrationeller Wanderungen.

Schon seit langem sind Bestrebungen im Gange, die immer noch stark verbreitete Einzelalpung auf Genossenschaftsalpung umzustellen. Einzelalpung, wie sie u. a. ur- 


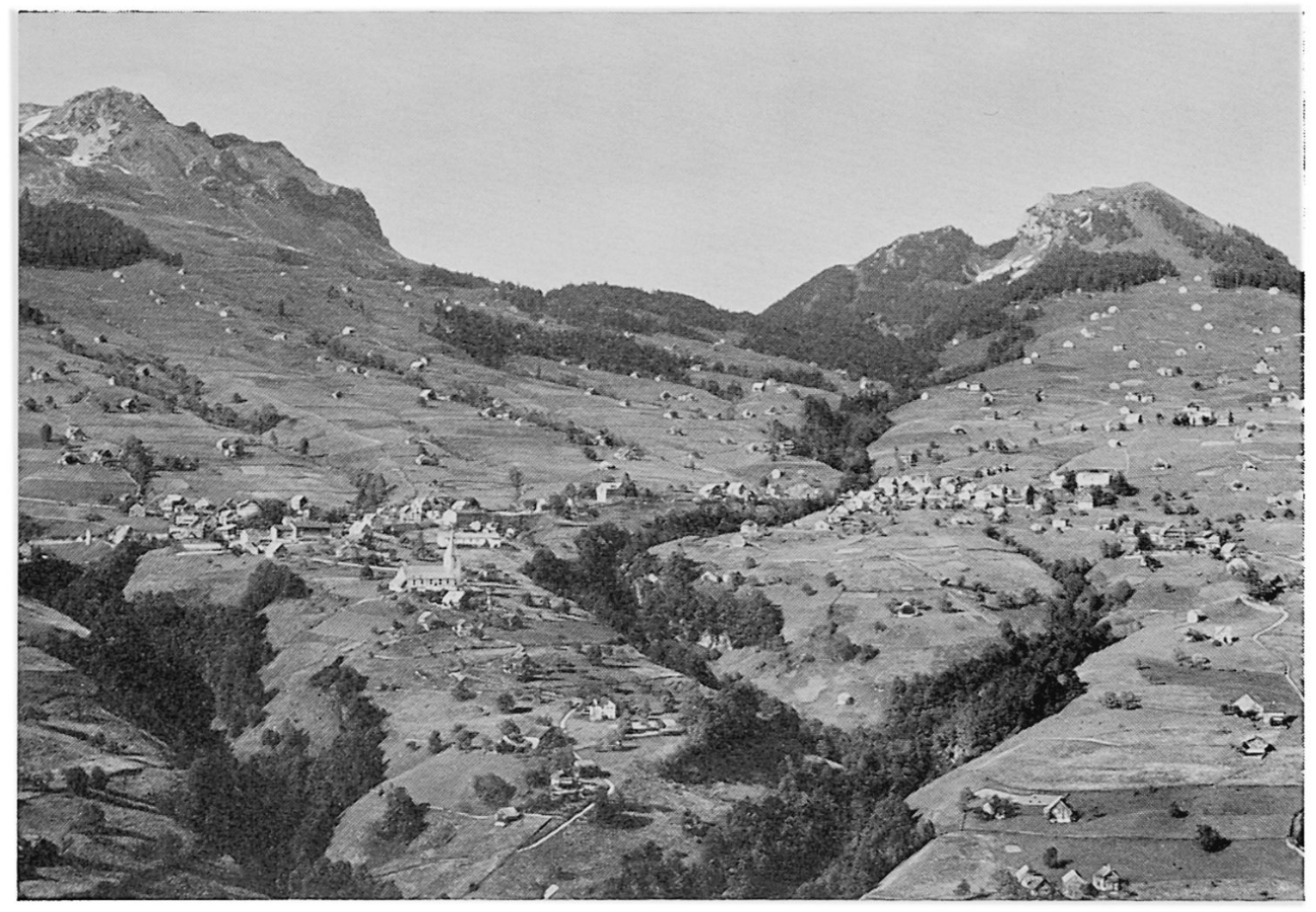

Abb. 1: Amden SG. Durch Bachtobel voneinander geschiedene Dorfteile, darüber Einzelhöfe und Alpweiden. Links der Mattstock, rechts der Gulmen.

sprünglich bei den Walsern üblich war, mag auch heute noch dort zweckmäßig sein, wo die Weidewege kurz sind, das heißt, wo das Heimgut relativ hoch und die Weide entsprechend nahe gelegen ist. So verläuft zum Beispiel die Alpgrenze des walserischen Weilers Juf (2126 m) im Avers GR, der höchstgelegenen Dauersiedlung der Schweiz, in unmittelbarer Nachbarschaft der Höfe. Das Vieh kann deshalb täglich aus den Ställen auf die nahe Weide geleitet und abends wieder zurückgetrieben werden. Maiensäße, die im allgemeinen übliche Stufe zwischen Heimgut und Alp, fehlen. Ähnlich sind die Verhältnisse in Amden SG. Die Vorteile aber fallen dahin, sobald die Distanzen zwischen Heimgut und Alpen groß sind und deshalb mindestens ein Familienglied auf der Alp weilen muß. Da die Gebäude bei Einzelalpung in Privatbesitz stehen, also auch vom Einzelnen unterhalten werden müssen, kann mit der Erstellung gemeinsamer Alphütten die wünschenswerte Konzentration und Rationalisierung erreicht werden. Auch auf den Weiden der Gemeinde Habkern BE besa $\beta$ früher jeder Genosse der «Bäuert», das heißt der lokalen Korporation, seinen eigenen Stall. Alle diese Ställe gruppierten sich in einem Alpdörfchen. In neuerer Zeit wurden manche dieser Ställe aufgegeben, dafür neue, größere und zweckmäßiger eingerichtete erstellt und nun gemeinsam verwendet. Man spart auf diese Weise Arbeitskräfte, Feuerungsmaterial, Gebäudeunterhalt, und man ist außerdem in der Lage, das Produkt, sei es Käse oder Butter, in besserer Qualität herauszubringen. Der gleiche Effekt wird erzielt, wenn auf einer sogenannten Kesselalp, das heißt auf einer Alp mit mehreren Senntümern, wie sie zum Beispiel im Glarnerland verbreitet sind, einzelne dieser Senntümer mit Nachbarsenntümern fusionieren. Weil jedes Senntum in der Regel mit eigener Sennhütte und eigenem Stall, oft auch mit je einem Schweinestall und einem Käsekeller ausgerüstet ist, führt die Konzentration sukzessive zur Verminderung der Hüttenzahl.

Der Einsparung von Alppersonal und technischen Einrichtungen und zugleich der qualitativen Verbesserung der Produkte dient u. a. auch die Konzentration der Käse- 
pflege im Tal von Etivaz (Château-d'Oex VD). 1935 wurde hier ein zentraler Käsekeller eingerichtet. Von etwa 30 Alpen der Umgebung werden die frischen Laibe hieher zur Einlagerung, fachmännischen Behandlung und zum Ausreifen gebracht und hernach in den Verkauf geleitet. Der Keller birgt bis zu 3000 große Gruyère-Käse und eine kleinere Zahl Saanen-Bergkäse. Das neuzeitlich ausgerüstete Gebäude steht an der Straße zum Col des Mosses, im Konvergenzpunkt zweier Nebentälchen, also in bester Verkehrslage, zugleich im funktionalen Zentrum der Talschaft mit seinen öffentlichen Diensten, wie Kirche, Schule, Post, Polizeistation, Milchsammelstelle und einigen generellen Gewerben. Das Abgeben eines bedeutenden Teils des Produktionsablaufs bringt den einzelnen Alpen die erwünschte Vereinfachung des Betriebes und, namentlich infolge des Verschwindens der eigenen Käsekeller, eine Veränderung der Alpsiedlung.

Ein weiterer positiver Beitrag zur Verbesserung der Betriebsform ist die Reduktion oder gar die völlige Aufhebung unrationeller Wanderungen. Wenn auch der übliche Wechsel von Mensch und Tier vom Talgut über das Maiensäß auf die Alp und wieder zurück durch das Relief bedingt und, sofern man die vorhandenen Gelände ausnützen will, durchaus zweckmäßig ist, so gibt es doch vielerorts komplizierte und nicht unbedingt nötige Wanderwege. $\mathrm{Zu}$ kleine und deshalb im Endeffekt defizitäre Stufen aufzugeben, ist daher ein verständliches Postulat. Dies gilt namentlich für die bekannten und viel beschriebenen Saisonwanderungen in einzelnen Walliser Tälern, insbesondere im Val d'Anniviers, wo sich der agrarische Wirtschaftsraum in der Vertikalen über rund $2300 \mathrm{~m}$ erstreckt und wo dieser breite Nutzungsgürtel nicht nur in drei, sondern in zahlreichere Etappen unterteilt und wo jede davon mit den nötigen Gebäuden ausgerüstet ist. Wenn auch die neuen Straßen diesen Saison-Nomadismus erleichtern, so sind die Wanderungen doch fast völlig zum Erliegen gekommen. Die Familien pflegen namentlich bei Erbgängen die einzelnen Stufen unter ihre Glieder aufzuteilen. Manche der auf diese Weise abgefundenen jungen Männer werden Fabrikarbeiter und betreuen das nun wesentlich kleinere Bauerngütchen nur noch im Nebenberuf. Ein Teil des in der Industrie bezogenen Barlohnes kann zur Verbesserung von Haus und Flur derartiger $Z$ wergbetriebe verwendet, auf diese $W$ eise die Landwirtschaft also aufgewertet werden.

\section{NEUVERTEILUNG DER AREALE}

Eine weitere Gruppe von Wandlungen besteht in der zweckmäßigeren Gliederung der Areale. Dies geschieht durch Neu-Unterteilung, durch Arrondierung oder auch durch einen Wechsel der Nutzungsform.

Im Bestreben, die Weide zu verbessern und ihre Nutzung zu intensivieren, schlägt man etwa ein Maiensä $\beta$ zur benachbarten Alp, verschiebt also die Alpgrenze in eine tiefere Lage. So wurden um 1928 im Val de Bagnes kleine Alpen zusammengelegt und die Weideflächen außerdem zu Lasten angrenzender Maiensäße vergrößert. Zusammengelegt wurden u. a. die Alpen La Lys und Sery ( $3 \mathrm{~km}$ südlich Lourtier VS). Sie erfuhren überdies eine zweckmäßige Vergrößerung durch Einbezug der darunterliegenden früheren Maiensäße Plana Dzeu, Le Tougne und Le Pissot (K. Suter). In ähnlicher Weise erweiterte man über Verbier gelegene Alpen zu Lasten angrenzender Maiensäße. Stets müssen im Zuge dieser Veränderungen die Alpgebäude der neuen Belegung durch Vieh angepaßt, also umgebaut oder durch neue Bauten ersetzt werden.

Manche Alp wird seit alters als große, zusammenhängende Fläche genutzt; eine Aufgliederung drängt sich auf. Wird nämlich die Fläche unterteilt, so läßt sich der Ertrag in der Regel heben, denn die einzelnen «Bezirke» können nun alternierend bestoßen werden, wobei das Gras der unbenützten Areale wieder nachwächst. Ging diese Aufgliederung früher nicht ohne Aufstellen von Zäunen, so bietet heute der leicht und rasch $\mathrm{zu}$ installierende Elektrohag eine billige und außerdem flexible Möglichkeit der Unterteilung. Aber auch eine vollständige Neuaufteilung früher größerer Weideareale 


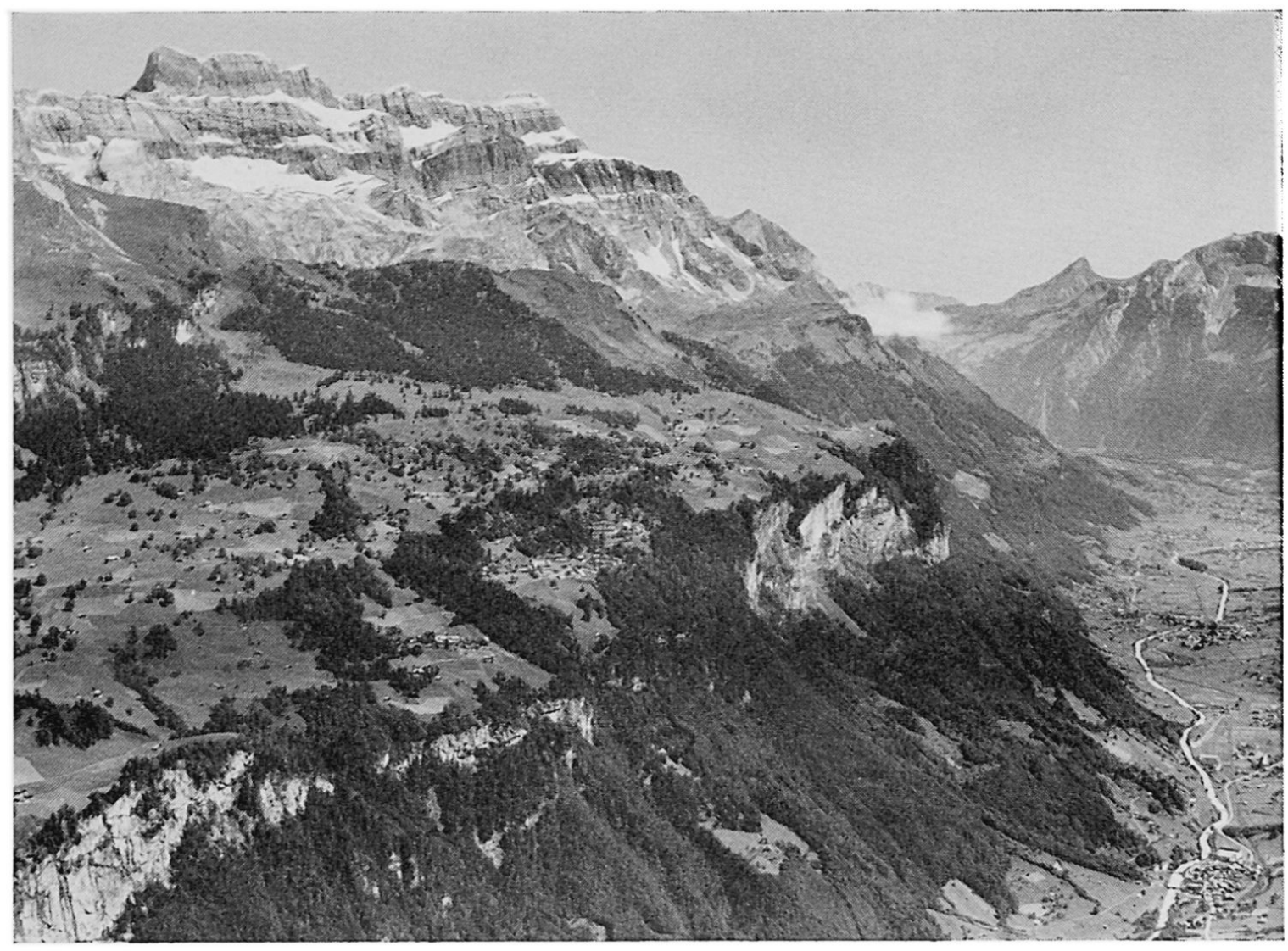

Abb. 2: Braunwald GL. Sonnige Bergterrasse in ungefähr $1300 \mathrm{~m}, 600 \mathrm{~m}$ über dem Linthtal Hotels und Ferienhäuser, bäuerliche Einzelhöfe, im oberen Teil Alpweiden.

erweist sich häufig als beste Lösung. So war die Bewirtschaftung der großen Korporationsalp Klewen ( $3 \mathrm{~km}$ südlich Beckenried NW) längst unbefriedigend. Den 28 beteiligten Alpbetrieben standen 29 Weiden mit 55 Alpgebäuden zur Verfügung, und zwar waren die meisten dieser Betriebe in zwei Stafel gegliedert, auf denen ein bis drei Sennten, je mit den zugehörigen Alpgebäuden lagen. Dieser Nutzungswirrwarr brachte selbstverständlich bedeutende Nachteile. Nun ist die Alp neu aufgeteilt und auf 25 arrondierte, einstafelige Betriebe reduziert, von denen jeder seine eigene Hütte hat. Baufällige Gebäude konnten aufgegeben, die Weiden gegeneinander abgehagt werden. Weitere Verbesserungen bestanden im Bau von Zufahrtswegen, Ausscheidungen von Streueland und Aufforstung. Die Sanierung änderte also Struktur und Physiognomie der Landschaft.

Eine derartige Arrondierung braucht sich natürlich nicht auf die Alpweiden zu beschränken; sie bringt unter Umständen noch nachhaltigeren Erfolg im Bereich der Heimgüter, also in Wiesen- und Ackergeländen. Hier kann sie durch die Abwanderung einzelner Familien eingeleitet und beträchtlich erleichtert werden. Denn die Zurückbleibenden werden in den meisten Fällen den frei werdenden Boden gerne durch Kauf oder in Pacht übernehmen und auf diese Weise ihren Besitz erweitern, vielleicht zugleich besser arrondieren. In vielen Fällen lohnt sich hernach der Einsatz landwirtschaftlicher Maschinen. Von derartigen Möglichkeiten wird begreiflicherweise oft Gebrauch gemacht, um so mehr, weil die Nachfrage von seiten der Zurückgebliebenen nicht besonders groß ist und die Preise deshalb erschwinglich sind. 
Eine dritte Gruppe von Verbesserungen läßt sich als technischer Ausbau bezeichnen. Hiebei ist an Gebäudesanierung, an den Bau von Seilanlagen und Milchleitungen sowie an die Motorisierung zu denken.

Es unterliegt keinem $Z$ weifel, daß sehr viele Wohnhäuser von Bergbauernfamilien den heutigen Ansprüchen an die Wohnkultur in keiner Weise mehr genügen. Die meisten dieser Gebäude sind überaltert, es fehlt überdies an zweckmäßigen Küchen, an Badezimmern, an hygienischen Einrichtungen und an den neuzeitlichen Haushaltgeräten. Glücklicherweise konnte in den letzten Jahren immerhin manches Haus verbessert werden, namentlich dort, wo es temporär an Feriengäste vermietet war. Die Bauernfamilie zieht im Hochsommer ins Maiensäß und überläßt ihre Wohnung fremden Besuchern. Eine solche Vermietung bringt eine willkommende Bargeldeinnahme. Wenn nur ein Teil dieses zusätzlichen Erwerbes zur Modernisierung der Einrichtung investiert wird, zum Beispiel zur Verbesserung der Küche, zum Einbau eines Badezimmers usw., so wird auf diese Weise die Wohnung merklich aufgewertet, so daß sie hernach leichter und teurer vermietet werden kann als vorher. Auf dem Urner Boden (Spiringen UR) haben die Korporationsbürger das Recht, eine Alphütte zu erstellen und $\mathrm{zu}$ unterhalten. Es gibt nun Bürger, die ihr Haus sukzessive ausbauten und an Feriengäste vermieten, dagegen ihr Weiderecht gar nicht mehr ausüben. Daß ein Fremder ein Ferienhaus erstellt, ist dagegen nicht möglich, die Korporation erteilt ihm hiefür kein Baurecht.

In unserer Zeit des Mangels an Alppersonal müssen die Alpbesitzer den wenigen, die sich für die Arbeit noch interessieren, bessere Lebens- und Arbeitsbedingungen bieten. Deshalb werden viele veraltete Alpgebäude saniert oder durch neue ersetzt. Es braucht heute gute Unterkunfts- und Schlafräume sowie zweckmäßig eingerichtete Sennküchen. Auch neue Ställe werden gebaut, alte soweit möglich verbessert. So steht zum Beispiel auf der Alp First, nördlich ob Grindelwald BE, für die Hirten eine besondere Wohnhütte, für die Rinder ein großes, in sechs Ställe unterteiltes neues Gebäude. Die Alp Lüser ( $5 \mathrm{~km}$ nordöstlich Engi GL) erhielt vor wenigen Jahren ein großes, neues Alphaus für zwei Senntümer, und zwar befinden sich im Mittelteil die zwei getrennten Sennküchen mit Alpstube im Erdgeschoß und Wohnkammern im Oberstock, gegen die beiden Enden des langen Gebäudes je zwei Doppelställe für 120 Kühe. Für den Fall, daß die beiden Senntümer einmal von einem einzigen Pächter übernommen werden sollten, können die beiden Sennküchen ohne weiteres zu einer einzigen verbunden werden.

Beträchtliche Verbesserungen bringen auch die Luftseilbahnen. Vielerorts, namentlich da, wo die Sommer- und Wintergäste zahlreich sind, wurden derartige Transportanlagen erstellt. Da die nötigen Bewilligungen von den Gemeinden gegeben, der Boden für die Stationen und für die Seilmasten von Korporationen und Privaten zur Verfügung gestellt wurden, mußten den Einheimischen fast stets Konzessionen in Form verbilligter Tarife eingeräumt werden. Aber auch für sich allein haben manche Bauern einfachste Seilanlagen erstellt, um die Bewirtschaftung ihrer Maiensäße und Alpen zu erleichtern. Auf solchen Anlagen werden Personen, Waren und Geräte in die Höhe transportiert, außerdem kann Heu abgeseilt, eventuell die Milch täglich in die Talsennerei zur Verarbeitung geführt werden. In Braunwald GL pflegen die Älpler etwa die Hälfte des anfallenden Mistes vom Oberstafel in den Unterstafel abzuseilen, weil er auf der unteren Stufe dank der klimatisch günstigeren Lage die Produktivität nachhaltiger zu fördern vermag als auf der oberen. Besonders zahlreich sind derartige Seilanlagen im Kanton Unterwalden, namentlich im Engelberger Tal. Die Gemeinde Oberdorf NW zählt allein 3 Personen- und 13 Warenseilbahnen. In Oberrickenbach (Wolfenschießen NW) soll es 300 Seile geben, davon sind 7 konzessionierte Transportbahnen für Personen. Bei der Scheune des Heimwesens Fur in Oberrickenbach laufen 7 
Heuseile zusammen, mit denen Heuballen von den obern Hängen herabgeführt werden. Auf dem Wiesenberg ob Dallenwil NW gab es früher nur Maiensäße; daß nun mehrere Familien dauernd hier oben wohnen und auch eine kleine Schule nötig wurde, ist allein auf den Bau einer leistungsfähigen Seilbahn zurückzuführen. Die Dauersiedlungsgrenze ist also hier höhenwärts gestiegen, eine große Ausnahme!

Für eine Reihe von hoch über den Talsohlen gelegenen Walliser Dörfern, wie Isérables, Unterbäch, Eischoll, war die Eröffnung einer Luftseilbahn der entscheidende Impuls für eine erfreuliche Entfaltung von Wirtschaft und Siedlung. Die Luftseilbahn ist billiger als eine Bergstraße, die in gewissen Gemeinden kaum je erstellt worden wäre. Nun bleiben junge Leute, die in der Industrie tätig sind, noch im elterlichen Bergbauernhaus und fahren täglich zu Tal an ihre Arbeitsstätte. Und wenn sie heiraten, können sie sich vielleicht ein eigenes Häuschen auf viel billigerem, weil von den Eltern zur Verfügung gestelltem Boden erstellen. 20 bis 40 Berufstätige aus den Dörfern Unterbäch und Eischoll werden von der Bahn täglich hinunter nach Turtig gebracht. Dies sind keine großen Zahlen, aber für die genannten Bergorte sind so viele zusätzliche Erwerbende mit Barlohn meist lebenswichtig. Mit Recht geben Kantone und Bund Beiträge an den Bau derartiger Anlagen. Sie betreiben damit eine aktive und offensichtlich sehr erfolgreiche Siedlungs- und Wirtschaftspolitik und treten damit der Entvölkerung der Bergtäler entgegen.

$\mathrm{Zu}$ den erfalgreichen technischen Verbesserungen gehört die Einrichtung von Milchleitungen, durch welche man die auf den Alpen gewonnene Milch direkt talwärts in die zentrale, modern eingerichtete und während des ganzen Jahres benützte Dorfsennerei führt. Statt dessen können die veralteten Sennküchen der Alp aufgegeben werden. Die Sennen bleiben ständig im Dorf, auf den Alpen sind nur noch Hirten und Melker stationiert. Die Konzentration und Stetigkeit der Verarbeitung fördert die Qualität der Produkte und ermöglicht zudem Einsparung an Personal. Die Sennen sind für die Arbeit in der schönen Dorfsennerei leichter zu gewinnen als für die abgelegene Alphütte. Mehrere derartige Milch-Pipelines sind u. a. in Walliser Bergtälern zur großen Zufriedenheit aller Beteiligten im Betrieb.

Noch weiter geht die Konzentration da, wo man weder auf der Alp noch in der Dorfsennerei Butter oder Käse herstellt, sondern die Milch entweder ohne weitere Verarbeitung als Konsummilch fortführt oder aber lediglich zentrifugiert, den Rahm ins Unterland verkauft. So wird es zum Beispiel am Fuße des Säntis, auf der Schwägalp, gehalten; der Rahm wird täglich nach Gossau SG geliefert, die Magermilch den Schweinen verfüttert. Auch in diesem Fall ist mit der betrieblichen Umstellung ein Funktionswandel von Gebäuden verbunden.

$\mathrm{Da} \beta$ die Motorisierung in Haus und Feld große Erleichterungen bringt, wurde bereits erwähnt. Sie kann Anlaß zum völligen Verzicht auf bisher benützte Gebäude geben. In den meisten Berggebieten setzt sich der Bodenbesitz eines Bauern aus an Steilhalden gelegenen Wiesen zusammen. Es bestehen also auch im Bereich des Heimgutes ausgeprochen ungünstige Reliefverhältnisse. Deshalb gehört zum Betrieb nicht nur eine in der Regel neben dem Wohnhaus stehende Scheune, sondern es gibt weitere in den Wiesenhängen verstreute Heuställe. Diese Verteilung war bisher zweckmäßig, weil man auf diese Weise das Heu und den Mist nicht weit zu tragen brauchte. Man nahm es in Kauf, daß im Winter mit der Viehhabe von Stall zu Stall zum Aufätzen des Heues gewandert und dafür die Milch täglich ins Heimgut gebracht werden mußte. Nun wurden in den letzten Jahren bestehende Wege vielerorts verbessert. In Fremdenorten, wie zum Beispiel in Braunwald GL, kam dieser Ausbau gleichzeitig den Feriengästen zugute. Die besseren Wegverhältnisse ermöglichen nun aber die Verwendung von Traktoren oder auch von einfachen Motorgefährten. Mit ihnen kann der Bauer sein Heu direkt in die Hauptscheune führen und also auf einzelne jener Heuställe verzichten. Die Folge ist, daß manche von ihnen sukzessive verschwinden, eine willkommene Einsparung an Gebäudeunterhaltskosten, die wiederum dem gesamten Betrieb zu- 
gute kommt. So ist es also namentlich der Motorisierung zuzuschreiben, daß die Zahl dieser Wirtschaftsgebäude sinkt.

Ganz ähnlich sind die Folgen auf vielen Bergwiesen. Manche Gemeinden und manche Alpgenossenschaft stellen ihren Bauern hochgelegene Bergwiesen-Parzellen (Heuwiesen, Heuberge) zur Verfügung, die je nach Produktivität nach je zwei oder drei Jahren oder sogar in längeren Intervallen genutzt werden. Das Bergheu pflegte man bisher entweder in Tristen aufzustapeln oder in kleine Holzschober zu versorgen und dann erst im Winter per Schlitten ins Heimgut zu bringen. Zahlreiche derartige «Schupperli» sind zum Beispiel im untersten Teil der Heualp Bischof (Elm GL), bei Bachghaltigen, zu drei Alpdörfchen vereinigt. Aber auch hier leiten Motorisierung und Seilanlagen den Wandel ein. Das Heu wird dank dieser Einrichtungen immer seltener an Ort aufbewahrt, sondern sofort nach dem Trocknen zu Tal geführt und auf dem Heustock versorgt. Die Heuhüttchen aber verschwinden nach und nach.

Am nachhaltigsten ist die technische Sanierung dort, wo sie sich nicht auf einzelne Betriebselemente beschränkt, sondern auf mehrere ausdehnt, wo also eine integrale Korrektur aufgezogen werden kann. Das ist vor allem in Gemeinden der Fall, denen die nötigen Gelder zur Verfügung stehen, so daß sie ihre vielfältigen öffentlichen Aufgaben in einer Weise zu lösen vermögen, die für die vielen andern, meist sehr ärmlichen Berggemeinden niemals möglich ist. Beispiele für derartige Integralmeliorationen gibt es namentlich im Wallis, wo Konzessionsgebühren, Wasserzinsen und Steuern der Kraftwerke zum Teil den beteiligten Gemeinden direkt zugute kommen. Der Kanton Wallis und das Kraftwerk Gougra bauten zum Beispiel 1957 die Talstraße des Val d'Anniviers großzügig aus, und in der Gemeinde Grimentz im besonderen konnten die meisten der alten Holzhäuser erneuert, die Güterzusammenlegung durchgeführt, eine neue Wasserversorgung, eine Irrigationsanlage, eine moderne Milchzentrale, Zimmer für Besuchsarzt und -krankenschwester eingerichtet werden. Anstelle der dem Stausee geopferten Alpweide schuf man durch Kauf von Nachbaralpen und Maiensäßen eine neue Alp Moiry und rüstete sie mit einem Stall für 200 Kühe und mit zentraler elektrischer Melkanlage aus. Also eine umfassende Neukonzeption und eine beachtliche wirtschaftliche Aufwertung. Ganz ähnlich und ebenso nachhaltig sind die Verbesserungen, welche die Gemeinde Hérémence VS dem Kraftwerk Grande Dixence zu verdanken hat (E. Schwabe).

\section{AUSBLICK}

Weil gegenwärtig im Mittelland ständig hochwertiges Agrarland durch Überbauung verlorengeht und sich die Menschen außerdem in unerwünschtem Maße in die Ballungsräume der Städte drängen, sollte der nötige Ausgleich zu diesen Vorgängen im Berggebiet geschaffen werden, wo sowohl noch Arbeitskräfte als namentlich auch freie Areale mit günstigen natürlichen Voraussetzungen zur Verfügung stehen.

In unseren Berggebieten bestehen zwei klar voneinander trennbare Entwicklungstendenzen: Einerseits gibt es Gegenden, in denen die Einwohnerzahl rückläufig ist, die Landwirtschaft immer extensiver, ja wo sogar bisheriges Agrarland samt den zugehörigen Wohnstätten aufgegeben wird. Daneben aber erfreuen sich andere Berggebiete eines unverkennbaren Aufschwunges, indem die Landwirtschaft saniert wird, neue Arbeitsplätze in Gewerbe und Industrie bereitgestellt und von Kraftwerken und vom Fremdenverkehr stammende Kapitalien für umfassende öffentliche Aufgaben investiert werden können.

Die große: Aufgabe besteht heute darin, auch die noch verbleibenden Notstandsgebiete der Alpentäler zu sanieren und Geld und Arbeit dort einzusetzen, wo begründete Hoffnung für eine erreichbare Aufwertung besteht. Es gilt also, sowohl das Ungleichgewicht zwischen Mittelland und Berggebieten als auch dasjenige zwischen verschiedenen Berggebieten unter sich zu beheben. Hiefür sind gezielte Maßnahmen vonnöten. 
Sie haben sich sowohl auf theoretische Kenntnisse als auch auf praktische Erfahrungen zu stützen. Das Studium alpiner Landschaften und die Anwendung landesplanerischer Prinzipien sind Voraussetzungen für den Erfolg.

\section{EINIGE LITERATUR}

Winkler, E.: Die Almgeographie in der Schweiz; in «Almgeographie», Forschungsberichte Nr. 4 der Deutschen Forschungsgemeinschaft. Mit ausführlichem Literaturverzeichnis. - Sonderheft «Landesplanung und Bergbauernfrage» des «Plan», Schweizerische Zeitschrift für Landes-, Regional- und Ortsplanung. Heft 4, 1959. - Die wirtschaftliche und soziale Lage der Schweizerischen Bergbevölkerung. Sonderheft der «Volkswirtschaft», Nr. 66, Bern 1959. — Gutersohn, H.: Geographie der Schweiz; Band II : Alpen, 1. Teil 1961, 2. Teil 1964.

\section{LA TRANSFORMATION ACTUELLE DES PAYSAGES CULTURELS ALPINS}

Alors que les villes et les villages du plateau suisse s'agrandissent rapidement, il existe des régions de montagne, d'où les jeunes s'en vont et où l'intensité d'utilisation des alpages diminue. Ce déséquilibre entre Plateau et zone alpine devrait être éliminé. D'autre part, bien des régions montagneuses présentent actuellement un développement positif, grâce aux activités des organisations officielles et privées, aux moyens financiers et aux possibilités de travail qu'apportent le tourisme, les usines électriques et les industries nouvellement implantées. Ce développement devrait être également appliqué aux régions montagneuses non privilégiées. Le succès de ces efforts dépend d'une étude géographique générale de la région et de l'utilisation des principes de planification.

\section{ALPINE ERHOLUNGSLANDSCHAFT}

\section{ERICH SCHWABE}

In den vergangenen Jahrzehnten, und seit dem Ende des letzten Krieges in besonders ausgeprägtem Maße, hat sich in bestimmten Teilen der Alpen ein tiefgreifender wirtschaftlicher Strukturwandel vollzogen. Wohl spielen die hergebrachten Beschäftigungen und Erwerbsarten der Bergbewohner - Land- und Alpwirtschaft, Holzgewinnung und -verarbeitung, Kleingewerbe - nach wie vor eine gewisse Rolle. Doch hat neben der primären, d. h. landwirtschaftlichen und bergbaulichen, und sekundären, verarbeitenden die sogenannte tertiäre, Dienste erbringende Produktion mit der Zeit beträchtliches Gewicht, zuweilen gar das Übergewicht erhalten. Die Funktion des alpinen Raumes erscheint mehr und mehr dahin ausgerichtet, zugereisten Bewohnern des Flachlandes Gelegenheit zur Erholung und Entspannung zu gewähren oder sie auf kurzfristiger Reise Eindrücke in sich aufnehmen zu lassen, die für sie neu sind. Der Fremdenverkehr hat sich damit zum nicht zu unterschätzenden Helfer der Bergbevölkerung in deren Daseinskampf entwickelt und der Abwanderung, der Land- und Bergflucht entgegenzuwirken vermocht. Seit seiner Erholung von der großen Krise der 30er Jahre hat er mächtig aufgeholt; seine modernen technischen Einrichtungen prägen über weite Strekken, von den Talsohlen bis in die Schneeregion hinauf, das Gesicht der Landschaft, und diesem Ausbau ist die unabläßige Vergrößerung und Schwerpunktbildung der Fremdenplätze parallelgegangen. Sie haben heute während bestimmter Abschnitte eines Jahres einer mehr oder minder beträchtlichen Agglomeration von ständigen und temporären Einwohnern zu dienen und sind zu eigentlichen zentralen Orten kleinerer, aber auch größerer Ordnung geworden.

Ein florierender Tourismus bietet, indem sich der sogenannte Multiplikatoreffekt geltend macht, u. a. der Landwirtschaft, den gewerblichen und kleinindustriellen Betrieben Einkünfte, die zum Teil deren Existenz überhaupt erst sichern. Für die direkte 